\title{
EXTENDED ULTRAVIOLET DISKS AND ULTRAVIOLET-BRIGHT DISKS IN LOW-MASS E/S0 GALAXIES
}

\author{
Amanda J. Moffett ${ }^{1}$, Sheila J. Kannappan ${ }^{1}$, Andrew J. BaKer ${ }^{2}$, and Seppo Laine ${ }^{3}$ \\ ${ }^{1}$ Department of Physics \& Astronomy, University of North Carolina, Phillips Hall, CB 3255, Chapel Hill, NC 27599, USA \\ ${ }^{2}$ Department of Physics \& Astronomy, Rutgers, the State University of New Jersey, 136 Frelinghuysen Rd., Piscataway, NJ 08854-8019, USA \\ ${ }^{3}$ Spitzer Science Center, California Institute of Technology, MS 220-6, Pasadena, CA 91125, USA \\ Received 2011 June 25; accepted 2011 October 26; published 2011 December 28
}

\begin{abstract}
We have identified 15 extended ultraviolet (XUV) disks in a largely field sample of $38 \mathrm{E} / \mathrm{S} 0$ galaxies that have stellar masses primarily below $\sim 4 \times 10^{10} M_{\odot}$ and comparable numbers on the red and blue sequences. We use a new purely quantitative XUV-disk definition designed with reference to the "Type 1" XUV-disk definition found in the literature, requiring UV extension relative to a UV-defined star formation threshold radius. The $39 \% \pm$ $9 \%$ XUV-disk frequency for these E/S0s is roughly twice the $\sim 20 \%$ reported for late-type galaxies (although differences in XUV-disk criteria complicate the comparison), possibly indicating that XUV disks are preferentially associated with galaxies experiencing weak or inefficient star formation. Consistent with this interpretation, we find that the XUV disks in our sample do not correlate with enhanced outer-disk star formation as traced by blue optical outer-disk colors. However, UV-Bright (UV-B) disk galaxies with blue UV colors outside their optical 50\% light radii do display enhanced optical outer-disk star formation as well as enhanced atomic gas content. UV-B disks occur in our E/SOs with a $42_{-8}^{+9} \%$ frequency and need not coincide with XUV disks; thus their combined frequency is $61 \% \pm 9 \%$. For both XUV and UV-B disks, UV colors typically imply $<1$ Gyr ages, and most such disks extend beyond the optical $R_{25}$ radius. XUV disks occur over the full sample mass range and on both the red and blue sequences, suggesting an association with galaxy interactions or another similarly general evolutionary process. In contrast, UV-B disks favor the blue sequence and may also prefer low masses, perhaps reflecting the onset of cold-mode gas accretion or another mass-dependent evolutionary process. Virtually all blue E/S0s in the gas-rich regime below stellar mass $M_{\mathrm{t}} \sim 5 \times 10^{9} M_{\odot}$ (the "gas-richness threshold mass") display UV-B disks, supporting the previously suggested association of this population with active disk growth.
\end{abstract}

Key words: galaxies: elliptical and lenticular, $\mathrm{cD}$ - galaxies: evolution - ultraviolet: galaxies

Online-only material: color figures

\section{INTRODUCTION}

In hierarchical models of galaxy formation, galaxies often experience mergers that result in early-type remnants. Disk structures are also predicted to regrow around some of these remnants (e.g., Steinmetz \& Navarro 2002; Governato et al. 2007), allowing for transitions back from early- to late-type morphologies. Observationally, a transition stage from late to early types, brought about by interactions, may be glimpsed in the population of E+A (post-starburst) galaxies (e.g., Yang et al. 2008). However, observational evidence for the opposite predicted transition, that from early- to late-type morphology, has remained more elusive.

The ultraviolet regime offers a natural choice for studying possible disk growth. Recently, GALEX has enabled the discovery of extended ultraviolet (XUV) disks (e.g., Thilker et al. 2005; Gil de Paz et al. 2005). These XUV disks show ongoing star formation beyond the optical radii and traditional star formation thresholds of late-type galaxies, providing an intriguing new look at galaxy disk growth in progress at $z \sim 0$. In a nearby galaxy sample emphasizing late types, Thilker et al. (2007, hereafter T07) find a $20 \%$ incidence of "Type 1" XUV disks, characterized primarily by large radial extents and structured UV morphologies (versus "Type 2" XUV disks, which consist of less extended UV-bright zones without morphological specifications).

GALEX has provided a useful platform for detection of star formation in early-type galaxies as well. Kauffmann et al. (2007) find that extended UV emission is common in high-mass bulge-dominated galaxies, likely associated with modest reservoirs of cold gas in the disk that help fuel bulge and black hole growth. Focusing specifically on galaxies with E/S0 morphology, extended UV emission has also been seen in ring structures around several S0 galaxies (Donovan et al. 2009; Cortese \& Hughes 2009) and Thilker et al. (2010) recently identified an XUV disk around the nearby S0 NGC 404. Salim \& Rich (2010) have also identified several $z<0.12$ early-type galaxies with extended UV structures in far-ultraviolet Hubble Space Telescope (HST) imaging.

The presence of XUV disks, however, can have a variety of interpretations. T07, for example, suggest an association of XUV disks with interactions or minor perturbations. The raw material for XUV-disk formation could be acquired externally from such interactions or from fresh cosmic gas accretion, either of which may be consistent with the extended disks and rings of H I commonly observed around E/S0s (e.g., Sage \& Welch 2006; Morganti et al. 2006; Oosterloo et al. 2007, 2010). Another possibility for creating extended disks in early types is the fallback of tidal tails in late-stage mergers (e.g., Hibbard \& Mihos 1995; Barnes 2002; Naab et al. 2006).

The evolutionary significance of disk growth may be greater in some of these scenarios than others. Of particular interest is the scenario of cold-mode gas accretion (e.g., Birnboim \& Dekel 2003; Kereš et al. 2005, 2009; Dekel \& Birnboim 2006), which may be linked to disk building in "blue-sequence E/S0s," a recently identified morphologically defined population of $\mathrm{E} / \mathrm{S} 0$ galaxies on the blue sequence in color versus stellar mass (Kannappan et al. 2009, hereafter KGB). Blue-sequence E/S0s 
are primarily found in non-cluster environments (KGB) and, as shown in KGB and Wei et al. (2010), many display global gas reservoirs and specific star formation rates that could allow the growth of significant new disks on relatively short timescales.

Cold-mode accretion occurs primarily below a critical shock heating stability mass (e.g., Birnboim \& Dekel 2003; Kereš et al. 2005); this mass may coincide with an observed "gas-richness threshold" stellar mass at $M_{\mathrm{t}} \sim 5 \times 10^{9} M_{\odot}$, below which blue-sequence E/S0s become suddenly common, along with gas-dominated galaxies (Kannappan 2004; Kannappan \& Wei 2008; see KGB regarding corrected mass scale). This low-mass regime may be where the most active $\mathrm{E} / \mathrm{S} 0$ disk growth occurs (KGB). Blue-sequence E/SOs also occur in modest numbers up to stellar masses of $\sim 3 \times 10^{10} M_{\odot}$, the bimodality mass of Kauffmann et al. (2003), above which classical spheroids with older stellar populations begin to dominate.

To better understand the significance of recent disk star formation in $\mathrm{E} / \mathrm{S} 0 \mathrm{~s}$, we concentrate on the mass regime up to the bimodality mass and seek to quantify the incidence of extended-disk star formation in a representative, largely field sample of E/S0s. In Section 2, we introduce our chosen sample and basic data. In Section 3, we discuss various methods for identifying extended star formation, adopting the T07 Type 1 XUV-disk designation as a reference. We then propose modifications to this definition to create a purely quantitative classification that reflects recent extended-disk star formation in early types. Since we are interested in the presence of disk star formation in a general sense, we also introduce an alternative UV-Bright (UV-B) disk definition, which can be used to identify significant disk star formation not necessarily extended relative to traditional star formation thresholds. In Sections 4 and 5 we present demographics and properties of our classified XUV and UV-B disks, and in Section 6 we compare our results to various formation scenarios and results from the literature. Finally, we provide a brief summary in Section 7 .

\section{SAMPLE AND DATA REDUCTION}

Our "GALEX GI" sample of $30 \mathrm{E} / \mathrm{S} 0$ s was defined for GALEX program GI3-0046 and primarily draws on the Nearby Field Galaxy Survey (NFGS; Jansen et al. 2000a). The sample was selected to encompass all of the NFGS blue-sequence E/S0s and the majority of NFGS red-sequence E/S0s in the stellar mass range below $\sim 4 \times 10^{10} M_{\odot}$ (Figure 1), where many E/S0s have substantial gas and settled blue-sequence E/S0s with the potential for disk regrowth are observed (KGB). The NFGS provides a representative sample of galaxies in the $z \sim 0$ universe with a wide range of luminosities, morphologies, and environments, allowing us to explore the natural variety of stages in galaxy evolution. In addition to $25 \mathrm{NFGS} \mathrm{E/S0s,} \mathrm{the} \mathrm{sample}$ includes 5 blue-sequence E/S0s from the "HyperLeda+" sample of KGB with comparable archival data.

To augment this sample, we have cross-matched all $M_{*} \lesssim$ $4 \times 10^{10} M_{\odot}$ E/SOs in the "HyperLeda+" sample of KGB with the GALEX and Spitzer archives to find sources imaged with exposure times similar to those for our prior programs. Excluding Virgo Cluster members from this cross-matched sample (consistent with the NFGS selection criteria), we find eight additional E/S0s for our "archival" sample.

Our primary data are GALEX NUV and FUV images at least as deep as those of the Medium Imaging Survey (MIS). For comparison of UV and optical morphologies, we employ DSS-II red images (http://archive.stsci.edu/dss/). For profile analysis, we compare to Spitzer IRAC $3.6 \mu \mathrm{m}$ imaging mostly

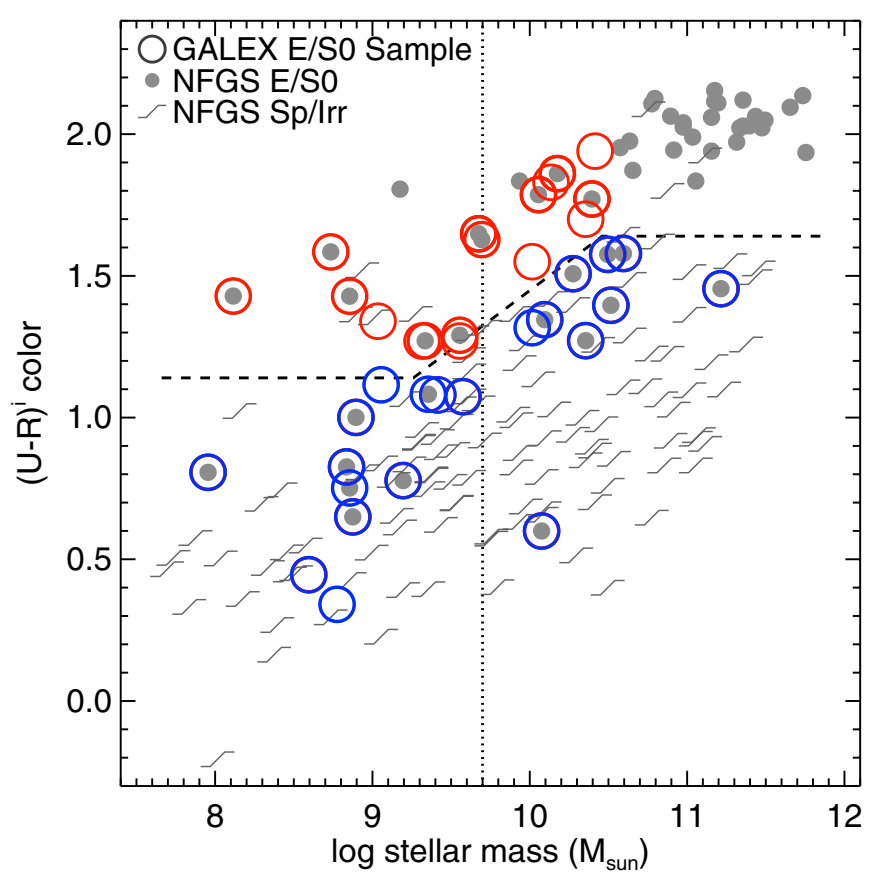

Figure 1. GALEX GI and archival E/SO sample in color-stellar mass space. The small gray symbols indicate galaxies in the Nearby Field Galaxy Survey, the parent sample for the majority of our E/S0s (Section 2). The dashed line divides the red and blue sequences, and the vertical line marks the gas-richness threshold mass (KGB). The $38 \mathrm{E} / \mathrm{S} 0$ s with $G A L E X$ data are denoted by open circles.

(A color version of this figure is available in the online journal.)

obtained for program GO-30406 with typical exposure times of $480 \mathrm{~s}$ (although several archival sources have exposure times down to $120 \mathrm{~s}$ ). The $3.6 \mu \mathrm{m}$ imaging serves as a proxy for $K$-band data, assuming the Leroy et al. (2008) conversion $I_{3.6}=0.55 I_{K}$ $\left(\mathrm{MJy} \mathrm{sr}^{-1}\right)$. We use the notation $K_{80}$ to denote the $80 \%$ light radius calculated using the $3.6 \mu \mathrm{m}$ data, to indicate the direct analogy with the $K_{80}$ radius of T07.

We use GALEX imaging in a pipeline-processed form with the zero-point calibrations of Morrissey et al. (2007). We apply foreground extinction corrections based on Schlegel et al. (1998) and Cardelli et al. (1989), but correction factors for internal extinction are not applied (consistent with prior XUVdisk studies). Spitzer IRAC $3.6 \mu \mathrm{m}$ imaging is also pipeline processed and calibrated according to procedures outlined in the IRAC Instrument Handbook (http://irsa.ipac.caltech.edu/ data/SPITZER/docs/irac/iracinstrumenthandbook/). In addition to the pipeline processing, we apply a median background subtraction procedure.

From these data, we extract radial surface brightness profiles and magnitudes by totaling fluxes in elliptical apertures. The parameters of these ellipses were determined from isophotal fits to optical images (as reported in Jansen et al. 2000a for NFGS galaxies) and newly derived using the IRAF ELLIPSE task and SDSS $g$-band imaging (Abazajian et al. 2009) for non-NFGS sample galaxies (parameters for non-NFGS galaxies in the GALEX GI sample from D. Stark et al., in preparation). Detection and masking of non-galaxy sources in these images were accomplished using SExtractor (Bertin \& Arnouts 1996). For calculation of comparative GALEX and Spitzer photometry, our UV and IR images were convolved with an appropriately sized Gaussian kernel to yield degraded images with the same point-spread function (PSF) FWHM as the lowest-resolution NUV images (FWHM 4".9). 
Table 1

Summary of Relevant UV-disk Definitions

\begin{tabular}{lll}
\hline \hline Definition & \multicolumn{1}{c}{ Extent Criterion } & \multicolumn{1}{c}{ Recent Star Formation Criterion } \\
\hline T07 Type 1 XUV & UV visually identified beyond $R_{\mathrm{UVSF}}$ & structured, bright UV with morphology different from optical \\
Purely Quantitative XUV & $\mathrm{UV}>3 \sigma$ above PSF shelf beyond $R_{\mathrm{UVSF}}$ & NUV $-K$ consistent with young population \\
UV-B & blue UV color beyond optical $R_{50}$ & NUV $-K$ consistent with $\gtrsim 10 \%$ young population \\
\hline
\end{tabular}

\section{IDENTIFYING EXTENDED STAR FORMATION}

Here, we discuss UV-based methods for identifying galaxies with recent star formation in disks and extended disks. Ideally, we seek to employ a purely quantitative method of classification. We also seek to answer two distinct questions about extended star formation in our sample, for which different specific identification methods are relevant. First, does it occur beyond traditional star formation thresholds? This question motivated the original "Type 1" XUV-disk definition of T07, which we take as a reference in designing a purely quantitative XUV-disk definition (Section 3.2). Second, is it significant (in a mass-contribution sense) in the optical outer disks of galaxies? This question motivates our introduction of a new UV-B disk definition (Section 3.3; see also Table 1 for a summary of definitions used in this paper).

We note that extension relative to UV-defined star formation thresholds does not necessarily imply extension beyond the full optical extent of the galaxy. Thus, another natural question about extended star formation is: does it extend beyond the optical galaxy? We will treat the answer to this question as a matter of investigation rather than definition, given that the radial extent of star formation relative to the optical disk may behave fundamentally differently in E/S0s versus late-type galaxies, for example in the case of inside-out disk (re)growth.

\subsection{Prior Definitions}

A natural choice for answering our first guiding question, concerning star formation extended beyond traditional star formation thresholds, is the T07 "Type 1" XUV-disk definition. T07 define Type 1 XUV disks as displaying more than one structured UV-bright emission complex beyond a centralized surface brightness contour corresponding to the expected star formation threshold (equated to an NUV surface brightness of $27.35 \mathrm{AB}$ mag $\operatorname{arcsec}^{-2}$ by $\mathrm{T} 07$, roughly matching typical $\mathrm{H} \alpha$ and $\mathrm{H}$ I thresholds; we label the corresponding radius $\left.R_{\mathrm{UVSF}}\right)$. In addition to extension relative to this UV contour, the definition requires that the XUV emission take on a different morphology from any underlying optical emission. T07 also define a Type 2 XUV-disk classification, but this is not geared toward tracing star formation beyond $R_{\mathrm{UVSF}}$, and an issue ${ }^{4}$ with the definition implies that we cannot apply it uniformly to early types. Thus, we do not consider Type 2 XUVs further here and henceforth are referring to Type 1 XUVs when we reference T07 XUV designations.

The T07 XUV definition is the basis for our new XUV definition (described in Section 3.2), but for completeness we note that several other measures of bright and/or extended

\footnotetext{
4 The Type 2 XUV-disk classification requires $\mathrm{FUV}(\mathrm{AB})-K(\mathrm{AB}) \leqslant 4$ in a large, optically low surface brightness zone within $R_{\mathrm{UVSF}}$ but outside $K_{80}$. Here "large" means an area at least seven times that enclosed within $K_{80}$. The Type 2 definition was developed for a late-type sample and has proved problematic to apply to E/S0s, in that $R_{\mathrm{UVSF}}$ often lies inside the $K_{80}$ radius, or lies outside but not as far as the definition requires (see also Moffett et al. 2010 for further details).
}

UV disks exist, most requiring high spatial resolution. For example, visual classification of UV structures such as rings is common in the literature (e.g., Cortese \& Hughes 2009; Salim \& Rich 2010; Marino et al. 2011). A quantitative variant on extended UV disk identification involves measuring individual UV knots in the outer regions of galaxies (e.g., Zaritsky \& Christlein 2007). Another quantitative approach lacking the high-resolution requirement is the blue integrated UV-color cut of Kauffmann et al. (2007). However, with an integrated color cut alone the correspondence between blue color and extended star formation is not necessarily one-to-one. We modify this approach by adopting an outer-disk UV color cut in our UV-B disk definition (see Section 3.3), addressing our second guiding question regarding significant star formation in the optical outer disks of galaxies.

\subsection{A New Purely Quantitative XUV-disk Definition}

To answer whether or not star formation occurs beyond $R_{\mathrm{UVSF}}$, we adopt the T07 XUV-disk definition as a useful reference definition and construct a purely quantitative alternative. Table 2 indicates the distribution and properties of the 16 XUVs we identify by the original T07 definition; see Figure 2 for an example. The primary criteria of the T07 XUV-disk classification are UV extension relative to $R_{\mathrm{UvSF}}$ and association of this emission with recent star formation. In the following sections, we discuss issues with these criteria that motivate elements of our modified definition, including consideration of possible UV upturn contributions and of the extended PSF shelf in the GALEX NUV.

\subsubsection{Ensuring Young Ages}

A possible concern in identifying XUV disks in E/S0s is the prevalence of the UV upturn, i.e., UV emission associated with old stellar populations (O'Connell 1999). To mitigate this issue, we identify XUV disks in the NUV (in contrast to T07's use of a combination of FUV and NUV data) since the UV upturn becomes stronger at FUV wavelengths. Nonetheless, 5 of the 16 XUVs we find using the original T07 XUV-disk definition have XUV-disk region FUV $-K$ colors red enough to be consistent with a $>1$ Gyr simple stellar population (SSP, as in T07 Figure 1).

In general, the T07 requirement that UV emission take on a different morphology from any underlying optical emission should preclude classifying an underlying old population as a separate XUV disk. However, the subjective requirement of structured emission can be difficult to apply consistently to samples like our own: our galaxies tend to have smaller angular sizes than those of T07, implying greater blurring at the low angular resolution of GALEX, so UV structure may be lost or be difficult to assess. An XUV-disk definition relaxing this requirement of structured emission has recently been applied by Lemonias et al. (2011) to a sample containing both early and late types, and they experiment with using an FUV $-r$ cut to ensure young populations.

Taking a similar approach but focusing on the NUV, we consider color cuts based on a suite of composite Bruzual 
Table 2

Sample Properties

\begin{tabular}{|c|c|c|c|c|c|c|c|c|c|c|c|c|c|}
\hline Galaxy & $\log \left(M_{*} / M_{\odot}\right)$ & Seq & Morph. & $\begin{array}{l}\text { Dist. } \\
(\mathrm{Mpc})\end{array}$ & $\log \left(M_{\mathrm{HI}_{\mathrm{I}}} / M_{\odot}\right)$ & $\begin{array}{l}\text { FUV-NUV } \\
\left(R>R_{\mathrm{UVSF}}\right)\end{array}$ & $\begin{array}{c}\mathrm{NUV}-K \\
\left(R>R_{\mathrm{UVSF}}\right)\end{array}$ & $\begin{array}{c}\mathrm{FUV}-\mathrm{NUV} \\
\left(R>R_{50}\right)\end{array}$ & $\begin{array}{l}\mathrm{NUV}-K \\
\left(R>R_{50}\right)\end{array}$ & T07 XUV? & XUV? & UV-B? & Sample \\
\hline NGC 3419 & 10.0 & B & $\mathrm{S} 0-\mathrm{S} 0 / \mathrm{a}$ & 43.4 & $9.1^{\mathrm{b}}$ & 2.9 & 5.0 & 2.5 & 5.1 & $\mathrm{Y}$ & $\mathrm{N}$ & $\mathrm{N}$ & GI \\
\hline NGC 4117 & 9.7 & $\mathrm{R}$ & So & 19.0 & 8.3 & 0.5 & 4.7 & 0.7 & 5.1 & $\mathrm{Y}$ & $\mathrm{Y}$ & $\mathrm{N}$ & GI \\
\hline NGC 3073 & 9.1 & B & $\mathrm{S} 0 / \mathrm{a}$ & 21.1 & 8.5 & 1.6 & 4.5 & 1.5 & 4.4 & $\mathrm{Y}$ & $\mathrm{Y}$ & $\mathrm{Y}$ & GI \\
\hline NGC 4308 & 8.7 & $\mathrm{R}$ & S0 & 8.4 & $<6.0$ & 3.1 & 5.4 & 2.3 & 5.6 & $\mathrm{Y}$ & $\mathrm{N}$ & $\mathrm{N}$ & GI \\
\hline IC 692 & 8.9 & B & $\mathrm{E}$ & 21.4 & 8.4 & 0.9 & 2.9 & 0.5 & 2.7 & $\mathrm{~N}$ & $\mathrm{~N}$ & $\mathrm{Y}$ & GI \\
\hline NGC 3011 & 9.4 & B & $\mathrm{S} 0 / \mathrm{a}$ & 25.7 & 8.3 & 0.8 & 3.3 & 0.4 & 3.4 & $\mathrm{~N}$ & $\mathrm{~N}$ & $\mathrm{Y}$ & GI \\
\hline NGC 3870 & 8.8 & B & Pec & 14.5 & 8.4 & 0.4 & 2.7 & 0.3 & 2.7 & $\mathrm{~N}$ & $\mathrm{~N}$ & $\mathrm{Y}$ & GI \\
\hline UGC 5923 & 8.1 & $\mathrm{R}$ & $\mathrm{S} 0 / \mathrm{a}$ & 8.0 & 7.7 & 1.1 & 3.8 & 0.7 & 3.6 & $\mathrm{~N}$ & $\mathrm{~N}$ & $\mathrm{Y}$ & GI \\
\hline NGC 5338 & 8.9 & $\mathrm{R}$ & So & 10.3 & 7.3 & 2.0 & 4.5 & 1.9 & 4.7 & $\mathrm{Y}$ & $\mathrm{Y}$ & $\mathrm{N}$ & GI \\
\hline IC 1141 & 10.4 & B & $\mathrm{S} 0 / \mathrm{a}$ & 68.0 & 9.3 & 0.7 & 3.5 & 0.6 & 4.0 & $\mathrm{~N}$ & $\mathrm{~N}$ & $\mathrm{Y}$ & GI \\
\hline IC 1144 & 11.2 & B & $\mathrm{S} 0 / \mathrm{a}$ & 175.4 & $<8.7$ & 2.3 & 5.8 & 2.3 & 6.0 & $\mathrm{~N}$ & $\mathrm{~N}$ & $\mathrm{~N}$ & GI \\
\hline IC 1639 & 10.6 & B & $\mathrm{cE}$ & 76.0 & 8.4 & 3.1 & 5.3 & 2.0 & 5.7 & $\mathrm{~N}$ & $\mathrm{~N}$ & $\mathrm{~N}$ & GI \\
\hline IC 195 & 10.5 & B & $\mathrm{S} 0 / \mathrm{a}$ & 52.1 & 9.4 & 1.9 & 6.2 & 1.9 & 6.2 & $\mathrm{Y}$ & $\mathrm{N}$ & $\mathrm{N}$ & GI \\
\hline UGC 9562 & 8.9 & B & So & 25.2 & 9.3 & 0.4 & 2.5 & 0.4 & 2.2 & $\mathrm{~N}$ & $\mathrm{~N}$ & $\mathrm{Y}$ & GI \\
\hline NGC 3032 & 9.6 & B & $\mathrm{Pec}$ & 25.2 & 8.3 & 2.0 & 4.7 & 1.8 & 4.8 & $\mathrm{~N}$ & $\mathrm{Y}$ & $\mathrm{N}$ & GI \\
\hline NGC 3522 & 9.7 & $\mathrm{R}$ & So & 22.9 & 8.4 & 2.1 & 4.9 & 2.0 & 5.1 & $\mathrm{Y}$ & $\mathrm{Y}$ & $\mathrm{N}$ & GI \\
\hline NGC 516 & 10.1 & $\mathrm{R}$ & S0 & 34.9 & $<7.4$ & 2.8 & 5.6 & 2.2 & 5.8 & $\mathrm{~N}$ & $\mathrm{~N}$ & $\mathrm{~N}$ & GI \\
\hline NGC 5173 & 10.3 & B & $\mathrm{E}$ & 41.2 & 9.3 & 0.8 & 3.9 & 0.7 & 4.2 & $\mathrm{Y}$ & $\mathrm{Y}$ & $\mathrm{Y}$ & GI \\
\hline NGC 5596 & 10.4 & $\mathrm{R}$ & So & 50.8 & 8.8 & 1.2 & 5.5 & 0.6 & 5.2 & $\mathrm{~N}$ & $\mathrm{~N}$ & $\mathrm{~N}$ & GI \\
\hline NGC 7077 & 8.8 & B & $\mathrm{S} 0 / \mathrm{a}$ & 18.9 & 8.2 & 2.1 & 3.1 & 0.7 & 3.0 & $\mathrm{Y}$ & $\mathrm{Y}$ & $\mathrm{Y}$ & GI \\
\hline NGC 7360 & 10.5 & B & $\mathrm{E}$ & 67.9 & 9.6 & 0.6 & 4.4 & 0.6 & 4.5 & $\mathrm{Y}$ & $\mathrm{Y}$ & $\mathrm{N}$ & GI \\
\hline UGC $12265 \mathrm{~N}$ & 10.1 & B & S0 & 82.8 & 9.4 & 1.1 & 4.7 & 1.0 & 4.8 & $\mathrm{~N}$ & $\mathrm{~N}$ & $\mathrm{~N}$ & GI \\
\hline UGC 6003 & 10.1 & B & $\mathrm{S} 0 / \mathrm{a}$ & 84.2 & 9.4 & 1.5 & 1.9 & 0.8 & 2.7 & $\mathrm{Y}$ & $\mathrm{Y}$ & $\mathrm{Y}$ & GI \\
\hline UGC 6570 & 9.6 & $\mathrm{R}$ & $\mathrm{S} 0 / \mathrm{a}$ & 28.6 & 8.4 & 1.4 & 4.5 & 1.0 & 4.6 & $\mathrm{~N}$ & $\mathrm{Y}$ & $\mathrm{N}$ & GI \\
\hline UGC 6637 & 9.2 & B & So & 31.5 & 8.6 & 0.8 & 3.7 & 0.3 & 2.9 & $\mathrm{~N}$ & $\mathrm{~N}$ & $\mathrm{Y}$ & GI \\
\hline UGC 6655 & 8.0 & B & S0 & 8.8 & 7.2 & 0.8 & 3.4 & 0.3 & 2.8 & $\mathrm{~N}$ & $\mathrm{~N}$ & Y & GI \\
\hline UGC 6805 & 8.9 & B & S0 & 20.3 & 7.6 & 1.3 & 3.9 & 0.6 & 3.5 & $\mathrm{~N}$ & $\mathrm{Y}$ & $\mathrm{Y}$ & GI \\
\hline UGC 7020A & 9.3 & $\mathrm{R}$ & So & 26.7 & 8.6 & 0.7 & 3.5 & 0.6 & 3.5 & $\mathrm{Y}$ & $\mathrm{Y}$ & $\mathrm{Y}$ & GI \\
\hline UGC 8876 & 10.2 & $\mathrm{R}$ & $\mathrm{S} 0 / \mathrm{a}$ & 36.7 & $<7.7$ & 1.6 & 6.3 & 1.5 & 6.4 & $\mathrm{Y}$ & $\mathrm{N}$ & $\mathrm{N}$ & GI \\
\hline NGC 3773 & 8.6 & $\mathrm{~B}$ & $\mathrm{Pec}$ & 10.5 & 7.9 & $\ldots{ }^{c}$ & 3.4 & $\ldots{ }^{c}$ & 3.2 & $\mathrm{~N}$ & $\mathrm{Y}$ & $\mathrm{Y}$ & GI \\
\hline IC 1024 & 9.4 & B & SOPec & 20.4 & $9.0^{\mathrm{b}}$ & 0.8 & 4.0 & 0.7 & 4.3 & $\mathrm{Y}$ & $\mathrm{Y}$ & $\mathrm{Y}$ & archival \\
\hline NGC 1047 & 9.0 & $\mathrm{R}$ & $\mathrm{S} 0 / \mathrm{a}$ & 19.1 & $8.7^{\mathrm{b}}$ & 2.3 & 5.3 & 2.5 & 5.2 & $\mathrm{~N}$ & $\mathrm{~N}$ & $\mathrm{~N}$ & archival \\
\hline NGC 2970 & 9.3 & $\mathrm{R}$ & $\mathrm{S} 0 / \mathrm{a}$ & 22.7 & $\ldots{ }^{a}$ & 2.4 & 5.2 & 2.0 & 5.2 & $\mathrm{~N}$ & $\mathrm{~N}$ & $\mathrm{~N}$ & archival \\
\hline NGC 3156 & 9.6 & $\mathrm{R}$ & So & 15.3 & $7.9^{\mathrm{b}}$ & 2.6 & 4.9 & 2.4 & 5.1 & $\mathrm{Y}$ & $\mathrm{Y}$ & $\mathrm{N}$ & archival \\
\hline NGC 3458 & 10.4 & $\mathrm{R}$ & So & 27.6 & $\ldots{ }^{a}$ & 1.9 & 6.2 & 1.7 & 6.5 & $\mathrm{~N}$ & $\mathrm{~N}$ & $\mathrm{~N}$ & archival \\
\hline NGC 4288A & 10.4 & $\mathrm{R}$ & So & 100.8 & $\ldots{ }^{a}$ & 1.4 & 6.1 & 1.1 & 5.9 & $\mathrm{~N}$ & $\mathrm{~N}$ & $\mathrm{~N}$ & archival \\
\hline NGC 5355 & 10.1 & $\mathrm{R}$ & S0/a-S0Pec & 34.4 & $9.5^{\mathrm{b}}$ & $\ldots{ }^{c}$ & 5.7 & $\ldots{ }^{c}$ & 5.8 & $\mathrm{Y}$ & $\mathrm{N}$ & $\mathrm{N}$ & archival \\
\hline NGC 5574 & 10.0 & $\mathrm{R}$ & $\mathrm{S} 0 / \mathrm{a}$ & 23.2 & $7.9^{\mathrm{b}}$ & 2.0 & 5.7 & 2.3 & 5.7 & $\mathrm{~N}$ & $\mathrm{~N}$ & $\mathrm{~N}$ & archival \\
\hline
\end{tabular}

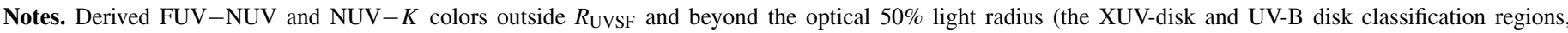

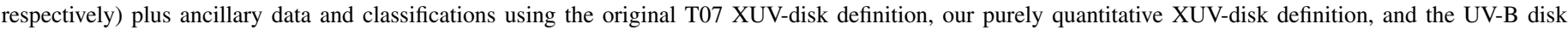
definition. Ancillary data are from Jansen et al. (2000a, 2000b), KGB, and Wei et al. (2010) except as noted. Distances assume $H_{0}=70 \mathrm{~km} \mathrm{~s}^{-1} \mathrm{Mpc}^{-1}$.

${ }^{\text {a }} \mathrm{H}$ I data unavailable.

${ }^{\mathrm{b}} \mathrm{H}$ I data from HyperLeda (Paturel et al. 2003).

c No FUV imaging available.

\& Charlot (2003) stellar population models using a Salpeter (1955) initial mass function, as described in KGB (see their Section 2.3). These composite models are built from two (young and old) components, with set age options, combined in a variety of ratios to create a large model grid. Similar to the grid of $\mathrm{KGB}$, the young SSP age options are 5, 25, 100, 290, 640, and 1000 Myr, while the old SSP age options are 1.4, 2.5, 3.5, .., 13.5 Gyr. The young SSP contributions can be $0 \%, 1 \%, 2 \%$, $4 \%, 8 \%, 16 \%, 32 \%, 64 \%$, or $100 \%$ of the population mass. SSP metallicities allowed in the grid are $Z=0.008,0.02$, and 0.05 . The young SSP can have 11 different extinction values, but here we consider only zero-extinction models for comparison to observed outer-disk colors. We make no explicit restriction on the metallicity combinations of the composite population models we consider, although we find that consideration of metallicity restrictions that could be reasonable in specific circumstances, such as $Z_{\text {young }} \leqslant Z_{\text {old }}$ or $Z \leqslant Z_{\text {solar }}$, do not substantially change the model color distributions we report (see Figures 3 and 4).

In one version of their XUV-disk classifications, Lemonias et al. (2011) used a color cut at FUV $-r=5$, designed to separate galaxies with recent XUV-disk star formation from those containing evolved populations (divider based on empirical red/blue-sequence division from Wyder et al. 2007). However, based on consideration of our stellar population model grid (Figure 3), this color selection can potentially exclude up to $\sim 30 \%$ of the composite populations with recently star-forming components.

Thus, we search for a different color selection that better encompasses composite stellar populations with young components. As a result of the aforementioned difficulties with using the FUV for this purpose and the practical usefulness of making such a selection in bands where data coverage is more complete, we prefer the NUV over the FUV. We find that NUV-based 

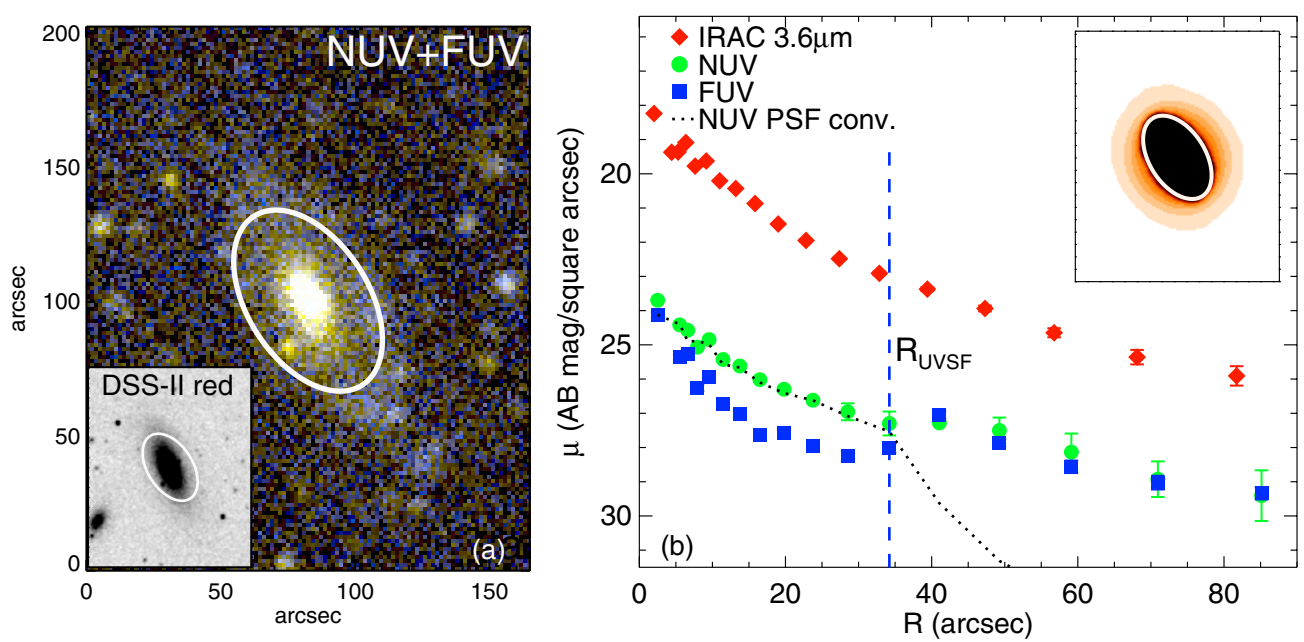

Figure 2. Images and surface brightness profiles of NGC 4117, one of several XUV-disk galaxies identified on the red sequence. (a) GALEX NUV+FUV color composite with overlay of the NUV-derived star formation threshold, $R_{\mathrm{UVSF}}$ (see Section 3 ). The inset shows the DSS-II red image with the same overlay for scale. (b) GALEX and Spitzer surface brightness profiles. A vertical line marks $R_{\mathrm{UvSF}}$. The black dotted line represents a profile extracted from the 2D (re)convolution of the NUV PSF with the NUV galaxy light within $R_{\mathrm{UVSF}}$ (see Section 3). The inset shows the (re)convolved image.

(A color version of this figure is available in the online journal.)

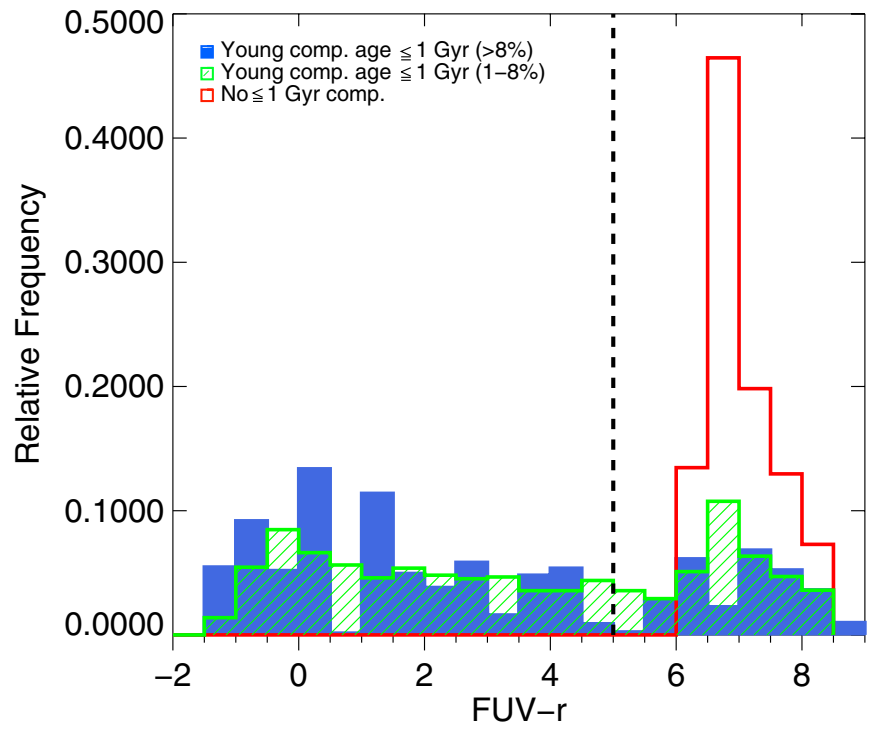

Figure 3. FUV $-r$ color for selected composite stellar population models (grid as described in Section 3.2.1), illustrating issues with using this color as a clean young/old population divider. Blue and green histograms represent numbers of models with $>8 \%$ and $1 \%-8 \%$ young population contributions by mass, normalized to the total numbers of such models. The red histogram represents numbers of models containing no young (age $\leqslant 1 \mathrm{Gyr}$ ) component, normalized to the total numbers of such models. The vertical dashed line indicates the color cut of Lemonias et al. (2011), which appears to miss a significant fraction $(\sim 30 \%)$ of the combined young model options that fall outside this cut with colors redder than FUV $-r=5$.

(A color version of this figure is available in the online journal.)

colors indeed display a more cleanly defined region where populations are predominantly old (compare Figures 3 and 4). From the model color distributions, it is apparent that the fraction of purely old models increases significantly beyond NUV $-K=5$, which is where young model fractions start to decline as well. Thus, we choose to exclude XUV disks with NUV $-K>5$.

\subsubsection{Ensuring Extended Emission}

When applying the original T07 XUV-disk definition, classifiers must subjectively identify the presence of extended

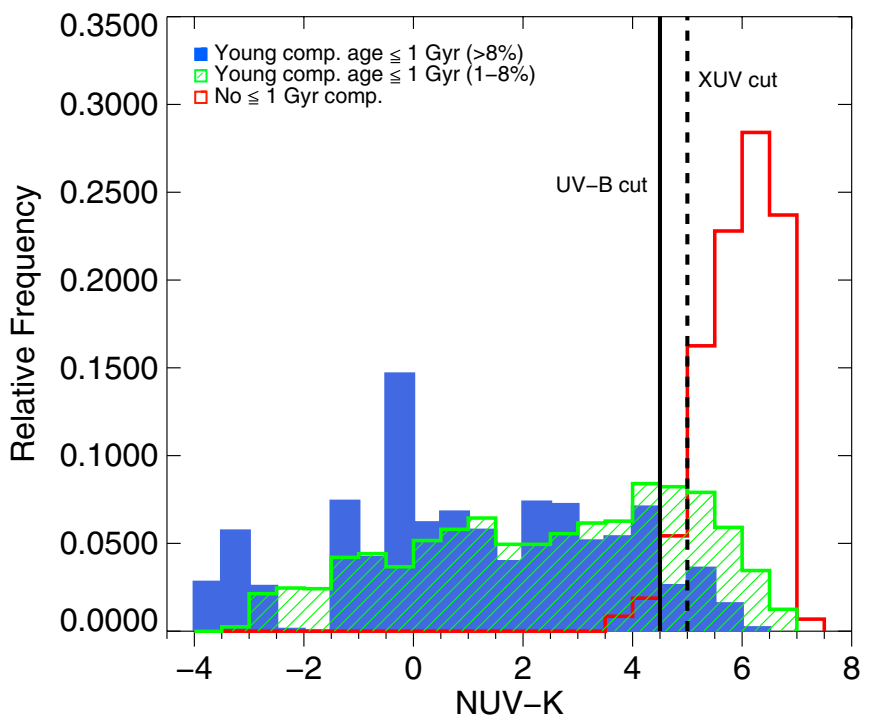

Figure 4. NUV $-K$ color for selected composite stellar population models (grid as described in Section 3.2.1), illustrating NUV $-K$ cuts chosen for our analysis. Blue and green histograms represent numbers of models with $>8 \%$ and $1 \%-8 \%$ young population contributions by mass, normalized to the total numbers of such models. The red histogram represents numbers of models containing no young (age $\leqslant 1 \mathrm{Gyr}$ ) component, normalized to the total numbers of such models. It is apparent that the fraction of purely old models increases significantly beyond $\mathrm{NUV}-K=5$, so we use this value to reject XUV disks likely to contain evolved populations as described in Section 3.2.1. A more conservative color cut at NUV $-K=4.5$ appears necessary if we wish to select populations with a significant young population as in our UV-B disk classification (here $>8 \%$, corresponding to the $\gtrsim 10 \%$ requirement specified in Section 3.3).

(A color version of this figure is available in the online journal.)

emission beyond $R_{\mathrm{UVSF}}$. However, when classifying XUVs from GALEX NUV imaging, especially when considering galaxies with small angular sizes, the $\sim 45^{\prime \prime}$ shelf in the NUV PSF (http://www.galex.caltech.edu/researcher/techdoc-ch5.html) may affect this judgement. Thus, to design a quantitative test for extension relative to $R_{\mathrm{UVSF}}$, we require that the NUV flux detected outside $R_{\mathrm{UVSF}}$ is significantly greater than ( $>3 \sigma$ above) the flux redistributed into this region by an artificial second convolution of the NUV PSF with the flux inside this radius. This 


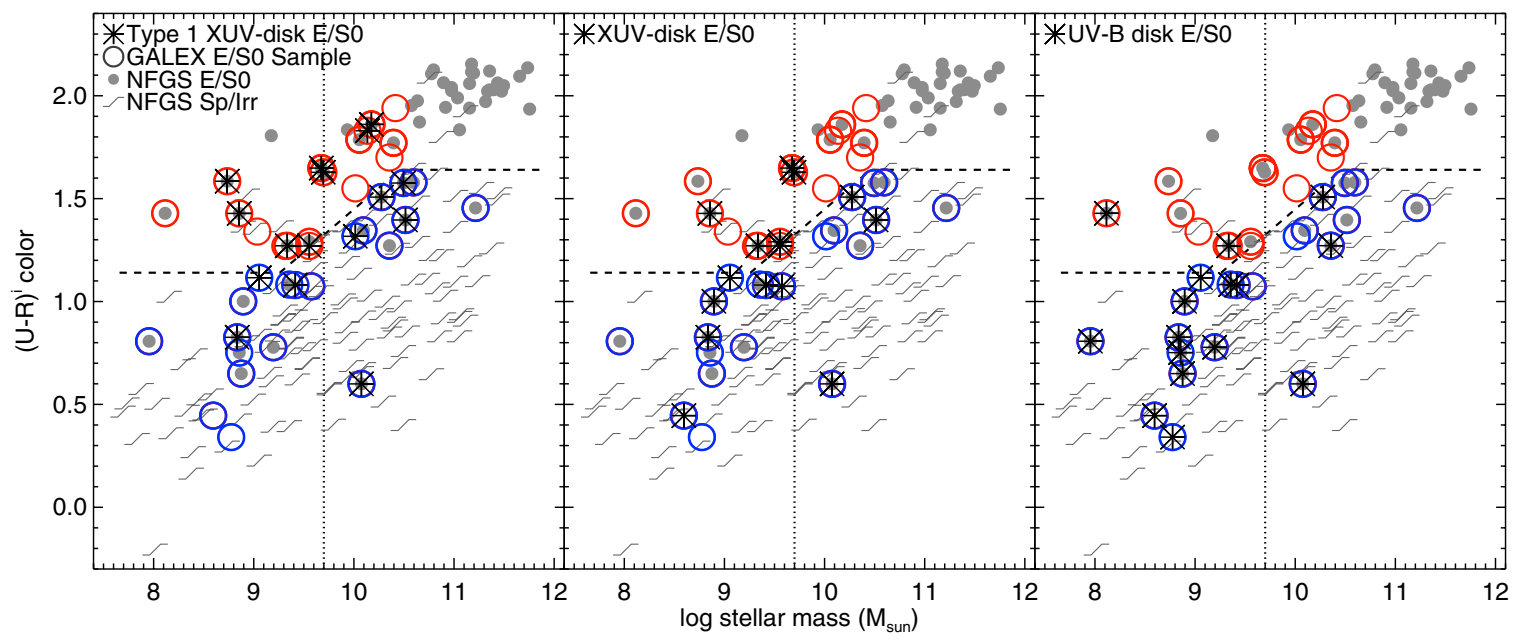

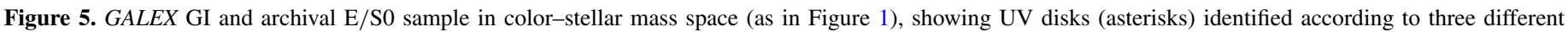
classifications: (left) the original T07 XUV-disk definition, (middle) our new, purely quantitative XUV definition, and (right) the UV-B disk definition.

(A color version of this figure is available in the online journal.)

(re)convolution is in addition to the natural convolution inherent in the images; see Figure 2 for an illustration. We note that for the XUV disks we have identified based on the original, subjective T07 definition, we have confirmed that this requirement is always satisfied.

\subsubsection{Final Definition}

In summary, for our "purely quantitative XUV-disk" designation, we ensure UV emission beyond $R_{\mathrm{UVSF}}$ by requiring $>3 \sigma$ emission above the NUV PSF shelf, and we ensure recent star formation by requiring NUV $-K<5$ in the XUV-disk region beyond $R_{\mathrm{UVSF}}$. Properties and demographics of these XUV disks are presented in Section 4 (see also Table 2) and largely imply that this population is associated with recent but not necessarily significant outer-disk star formation.

With our new definition, we identify a similar fraction of XUV disks as when applying the traditional T07 Type 1 XUV-disk definition (see Table 2, Figure 5), but the overlap between these classifications is not perfect. Approximately $70 \%$ of the traditionally identified XUVs are among XUVs identified with our purely quantitative method. In cases where the classifications do not overlap, the reason is either (1) insufficiently blue NUV $-K$ color to satisfy the new definition's color cut or (2) UV disk morphology not distinct enough from the optical to satisfy the T07 Type 1 definition. Our color cut is more conservative in rejecting XUV disks that may contain evolved populations than the T07 requirement of morphological differences compared to the optical. On the other hand, the T07 morphology requirement may recover XUVs with even weaker or more incipient star formation than our definition allows, where this star formation has not built up a detectable optical counterpart.

\section{3. $U V$-Bright $(U V$-B) Disk Definition}

To answer whether or not significant UV-detected star formation occurs in the optical outer-disk region, irrespective of extent beyond $R_{\mathrm{UVSF}}$, we construct a second quantitative classification.

We designate a population with $\mathrm{a} \gtrsim 10 \%$ young component by mass as one containing "significant" star formation (in practice for our model set $>8 \%$, Section 3.2.1). Considering the aforementioned stellar population model grid, a more conservative color cut than was used in the purely quantitative XUV case appears necessary to select galaxies containing significant recent star formation (Figure 4). Requiring NUV $-K<4.5$ presents a natural choice for this definition, given the falloff in the fraction of models with $\mathrm{a} \gtrsim 10 \%$ young population component beyond this value.

To quantify our region of interest for this definition, i.e., the optical outer disk, we select the region beyond the optical $50 \%$ light radius. Thus, our UV-B disk classification requires only NUV $-K<4.5$ beyond the optical $50 \%$ light radius. The properties and demographics of the UV-B disks are presented in Section 5 (see also Table 2), from which we conclude that these galaxies correlate well with enhanced optical disk star formation.

\section{XUV-DISK PROPERTIES AND DEMOGRAPHICS}

With our purely quantitative definition, we identify XUV disks in $15 / 38$ or $39_{-9}^{+9} \%$ of our E/S0 sample (see Table 2 for the identifications and Figures 8-10 for images of classified XUVs and non-XUVs). These XUV-disk classifications supersede the preliminary, purely visual classifications of Moffett et al. (2010), which were made without reference to $R_{\mathrm{UvSF}}$. In the following, we present the demographics and basic properties of the identified XUVs.

\subsection{Extents and Ages}

The XUV disks in our E/S0s can extend beyond $R_{25}$, as has been found in Type 1 XUV disks for late types (e.g., Thilker et al. 2005; Gil de Paz et al. 2005; T07; see also Zaritsky \& Christlein 2007). We find radial extents (to the last measured NUV point) $\sim 0.7-2.3 R_{25}$, with mean $\sim 1.3 R_{25}$ and $\sim 70 \%$ extending beyond $R_{25}$. Relative to the older populations traced by near-IR light, the average radial extent of the young XUV-disk component in our E/S0s is $\sim 2$ times the $K_{80}$ radius. Relative to the centralized younger populations traced by NUV light, the average radial extent of our XUV disks is $\sim 1.5$ times $R_{\mathrm{UVSF}}$.

Compared to XUV disks in late-type galaxies, our E/S0 XUV disks tend to be redder. The reported outer-disk FUV-NUV colors of late-type XUV-disk galaxies in the literature range primarily between small negative values and $\sim 0.5$ (Thilker et al. 2005; Gil de Paz et al. 2005, 2007). Our early-type 


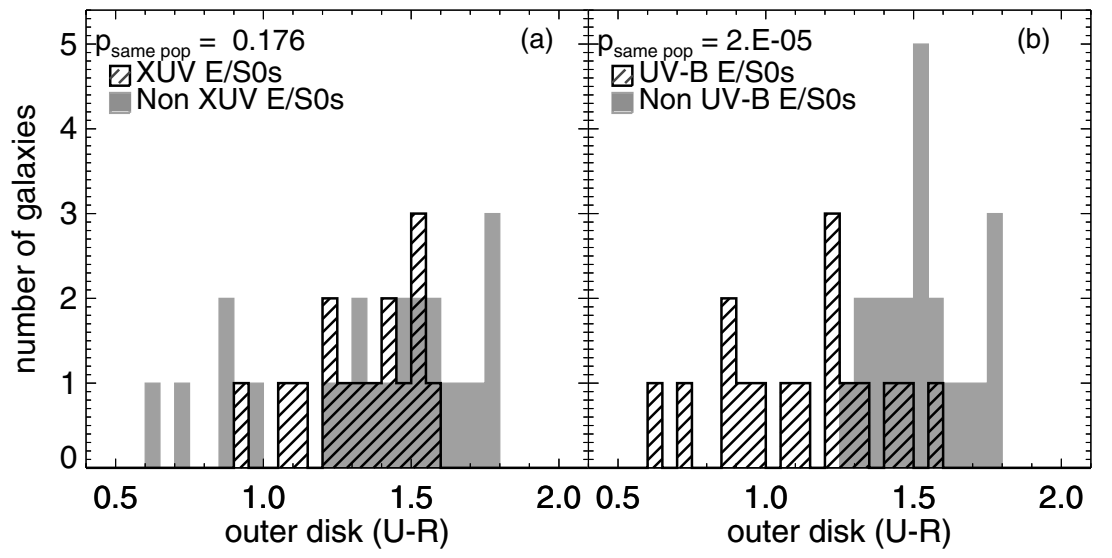

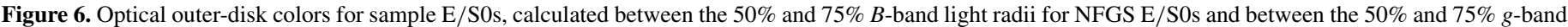

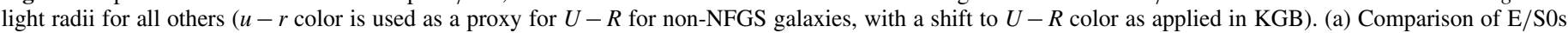

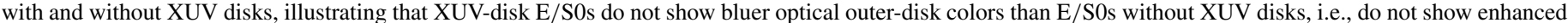

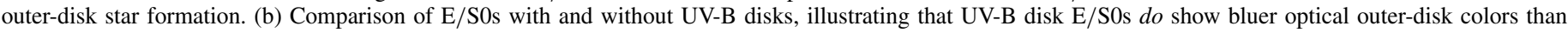
E/S0s without UV-B disks, i.e., do show clearly enhanced outer-disk star formation.

XUV-disk galaxies have an average color of $\sim 1.4$ in the XUV-disk regions (similar to the early-type XUV-disk galaxy NGC 404; Thilker et al. 2010). However, the contour at $R_{\mathrm{UVSF}}$ for our early-type XUVs tends to occur closer to $K_{80}$ than it does for late-type XUVs (enclosing on average $\sim 3$ times the area of the $K_{80}$ contour versus $\sim 15$ for late types; see T07). Thus, redder XUV-disk colors in early types may simply indicate a greater contribution from the underlying old stellar population than is typical for late types.

The XUV-disk FUV-NUV colors we compute for our $\mathrm{E} / \mathrm{S} 0$ s are consistent with $<1$ Gyr ages from SSP models. We choose to report SSP-equivalent ages for our XUVs in light of the inherent degeneracies involved in estimating separate old/young population ages from composite population models. We note that age estimates from stellar population models are affected by uncertainties in modeling the UV contribution from old stellar populations and will also vary depending on the assumed star formation history. Comparing with Bruzual \& Charlot (2003) UV model colors for an instantaneous starburst with $Z=0.02$ (as in T07 Figure 1), our average XUV-disk FUV-NUV color of $\sim 1.4$ corresponds to an SSP with an approximate age of 500 Myr.

One of our XUV-disk galaxies does have XUV-disk region FUV $-K$ color red enough to be consistent with a $>1$ Gyr SSP (as in T07 Figure 1). However, all of our XUV-disk galaxies, including this red FUV $-K$ case, display independent indicators for recent or potential star formation, in the form of either $\mathrm{H} \alpha$ or $\mathrm{H}$ I detections in the NFGS or the literature.

\subsection{Demographics}

We find XUV disks in both red- and blue-sequence E/S0s and over a wide range in stellar mass (Figure 5). On the red sequence, the XUV-disk frequencies are $0^{+23} \%$ and $60_{-20}^{+18} \%$ above and below the gas-richness threshold mass (at stellar mass $\left.M_{\mathrm{t}} \sim 5 \times 10^{9} M_{\odot}, \mathrm{KGB}\right)$, respectively. On the blue sequence, the corresponding frequencies are $33_{-18}^{+22} \%$ and $50 \% \pm 18 \%$.

If we ignore mass dependence, we find no clear evidence for a preference in XUV-disk incidence between redand blue-sequence E/SOs. Assuming a probability for an XUV-disk galaxy to be on the blue sequence equal to the overall sample blue-sequence fraction, binomial statistics yield a $46 \%$ probability of obtaining at least the number of XUV-disk galax- ies observed on the blue sequence out of the total number of XUVs identified.

Likewise, if we ignore sequence dependence, we find that the XUV-disk galaxy stellar mass distribution is not significantly different from that of the parent E/S0 sample $(61 \%$ probability of being drawn from the same distribution in a Kolmogorov-Smirnov test). Binning the data in mass yields a hint of a difference: the frequencies of XUV disks are $19_{-10}^{+15} \%$ and $55_{-13}^{+12} \%$, respectively, above and below $M_{\mathrm{t}}$, although the significance of this difference is not high $(\sim 1.8 \sigma$ confidence $)$.

We note that XUV disks identified according to the original T07 definition have an even more uniform color/mass distribution (Figure 5). Considering the slope of the color-stellar mass sequences, this difference is consistent with what one might expect as a consequence of our purely quantitative XUV definition excluding XUV disks with the reddest colors.

\subsection{Star Formation}

Although our XUV disks reflect recent star formation, we find that they do not show substantial recent star formation as traced by blue optical outer-disk colors (Figure 6). Likewise, the $\mathrm{E} / \mathrm{S} 0$ s with XUV disks do not show enhanced atomic gas content relative to the E/S0s without XUV disks, instead yielding a $36 \%$ Kolmogorov-Smirnov test probability of the same $M_{\mathrm{HI}} / M_{*}$ distribution (Figure 7). These possibly counterintuitive results imply that XUV disks are not necessarily associated with strong star formation and may instead be associated with weak/incipient star formation due to a process affecting the galaxy population broadly, an idea that we return to in Section 6.

We note that the T07 requirement of different UV-optical morphology may pick out weak star formation to an even greater degree than our purely quantitative approach, since the UV-optical morphology difference could imply that the UV-detected star formation is not substantial or sustained enough to have built up an optical counterpart.

\section{UV-B DISK PROPERTIES AND DEMOGRAPHICS}

With the UV-B disk classification, we identify $16 / 38$ or $42_{-8}^{+9} \%$ of our sample as UV-B disks (see Table 2 for identifications, Figures 8 and 9 for images of classified UV-Bs). Although we find similar frequencies of XUV and UV-B disks in our sample, and about half of the galaxies with UV-B disks 


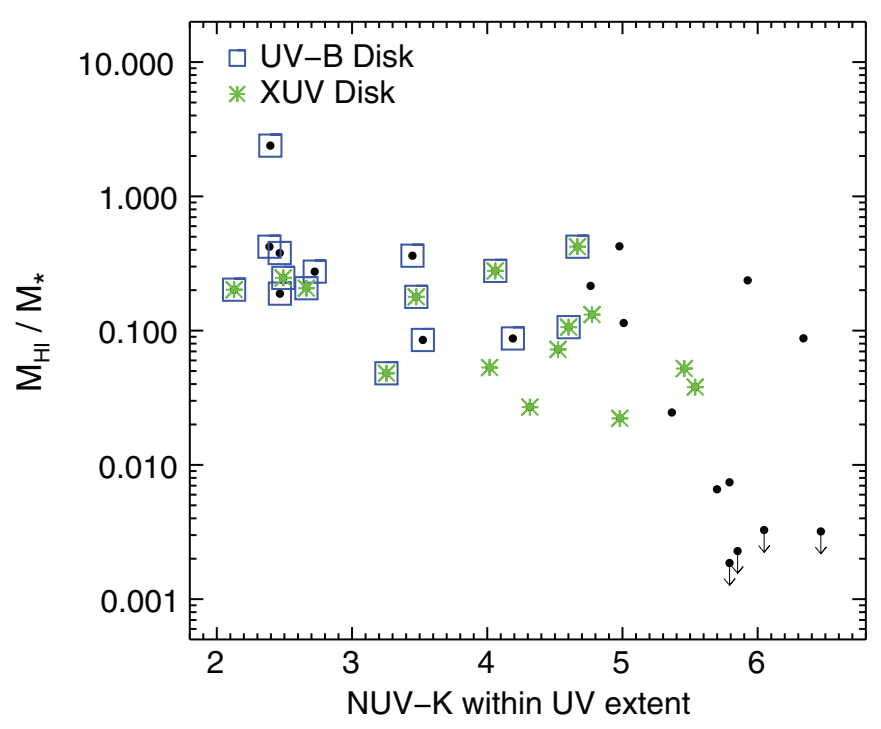

Figure 7. $\mathrm{H}$ I content vs. NUV $-K$ color measured within the detected NUV extent, illustrating the trend toward enhanced $\mathrm{H}$ I content in UV-B disk galaxies (blue squares). Black points represent all sample galaxies with $\mathrm{H}$ I data $\left(M_{\mathrm{H}}\right.$ from references noted in Table 2) and green stars represent XUV disks. Note that the plotted NUV $-K$ colors are not those used for classification of either XUV or UV-B disks.

(A color version of this figure is available in the online journal.)

also host XUV disks, the overall properties and demographics of these two classes display a number of differences, as we discuss in the following sections.

\subsection{Extents and Ages}

Similar to our quantitatively identified XUV disks, the UV-B disks we identify typically extend beyond $R_{25}$, with an average extent (to the last detected NUV point) of $\sim 1.4 R_{25}$ and all extending beyond $R_{25}$. The average UV-B disk extent relative to the near IR is slightly larger than for XUV disks at $\sim 2.3 K_{80}$ while the average extent relative to the UV is smaller than for XUV disks at $\sim 1.3 R_{\mathrm{UVSF}}$.

The UV-B disk FUV-NUV colors we observe are also consistent with $<1$ Gyr SSP ages. For UV-B disk galaxies, the average FUV-NUV color outside the optical 50\% light radius is $\sim 0.6$, which corresponds to a slightly younger $\sim 300 \mathrm{Myr}$ SSP-equivalent age than is found for XUV disks. No UV-B disks display $\mathrm{FUV}-K$ colors red enough to imply SSP ages older than 1 Gyr.

\subsection{Demographics}

In contrast to the widespread distribution of XUV disks, UV-B disks are preferentially found on the blue sequence and may prefer the low-mass regime as well (see color-mass distribution in Figure 5).

If we ignore mass dependence, we find clear evidence for a preference in UV-B disk incidence between red- and blue-sequence E/SOs. Assuming a probability for a UV-B disk galaxy to be on the blue sequence equal to the overall sample blue-sequence fraction, binomial statistics yield a low $0.7 \%$ probability of obtaining at least the number of UV-B disks observed on the blue sequence out of the total number of UV-Bs identified.

If we ignore sequence dependence, we find that the UV-B disk galaxy and full sample stellar mass distributions have an $8 \%$ Kolmogorov-Smirnov test probability of being drawn from the same distribution, which implies they are not conclusively distinct. Similarly, the UV-B disk frequencies we calculate are $19_{-10}^{+15} \%$ and $59_{-13}^{+12} \%$ above and below $M_{\mathrm{t}}$, respectively, which are more different than in the XUV-disk case, but still only distinct at approximately $2 \sigma$ confidence.

\subsection{Star Formation}

In contrast to XUV disks, the UV-B disks in our sample do correlate with elevated star formation as traced by blue optical outer-disk color (Figure 6). E/S0s with UV-B disks also show enhanced $\mathrm{H}$ I content relative to E/S0s without UV-B disks (Figure 7), with $0.1 \%$ Kolmogorov-Smirnov test probability of the same $M_{\mathrm{HI}} / M_{*}$ distribution. Thus, it appears that UV-B disks are closely linked to significant star formation potential and pronounced optical outer-disk star formation.

\section{DISCUSSION}

In this section, we compare our identified XUV and UV-B disk galaxy properties and demographics to XUV-disk and early-type galaxy formation scenarios and related literature results. We note, however, that uniform knowledge of the local and global environments of our sample galaxies would be necessary to constrain these formation scenarios and that uniform environmental data are not available for our sample. Thus, study of the environmental properties of such galaxies is deferred to future work.

\subsection{High Frequencies of $X U V$ and $U V$-B Disks}

XUV and UV-B disks occur in our sample with individually high, approximately $40 \%$ frequencies, and a combined frequency of $61 \% \pm 9 \%$. Compared to classical "red and dead" expectations for early-type galaxies, the high incidence of apparent extended star formation we observe in XUV disks, with $\sim 70 \%$ extending beyond the optical $R_{25}$, is in itself a surprising result and may provide evidence against $\mathrm{E} / \mathrm{S} 0$ formation through quenching processes in the low-mass, largely field regime we sample. Moreover, that we observe a similarly high incidence of UV-B disks, which seem to relate more closely to significant star formation and that all extend past $R_{25}$, is even more remarkable. In addition, although differences in samples and definitions complicate comparisons of absolute XUV-disk frequencies, it is intriguing that we find a frequency approximately twice the $\sim 20 \%$ reported in late types by T07 (see also Lemonias et al. 2011).

One possible explanation for the high incidence of XUV disks we observe in E/SOs could be a formation channel that involves mergers. Fallback of tidal tails in the late stages of a merger that is major enough to produce a spheroid is a likely scenario for creating new extended disks (e.g., Barnes 2002; Naab et al. 2006). Early-type galaxies at low luminosities/masses are largely "fast rotators" (as per the Emsellem et al. 2007 terminology), displaying disk-like dynamics reflecting the importance of gas in mergers related to their formation (e.g., Davies et al. 1983; Emsellem et al. 2007; KGB). Thus, if such mergers often form XUV disks, the high frequency we observe in our mass regime could be a natural consequence.

Another possible explanation for the high XUV-disk incidence in early types compared to late types, related to the inferred weak nature of XUV-disk star formation (Section 6.3), could be a bias due to the relative ease of detecting small star formation events in E/S0s. Such events may have a more detectable 


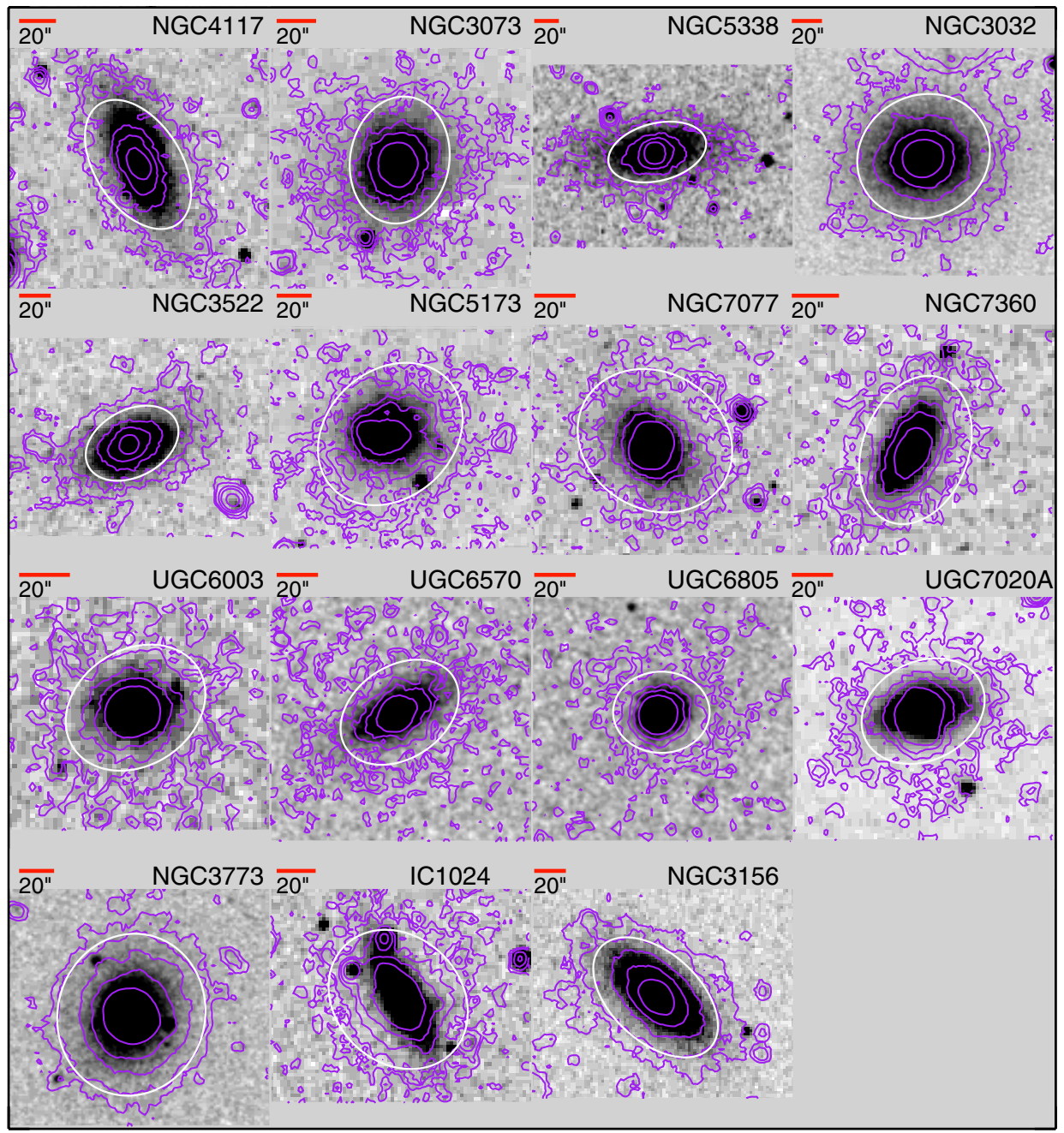

Figure 8. Smoothed NUV contours (purple) overlaid on DSS-II red images of XUV-disk E/S0s. $R_{\mathrm{UVSF}}$ is indicated in white, and contours start at $\sim 28.6$ AB mag $\operatorname{arcsec}^{-2}$ and go up by $2 \times, 5 \times, 10 \times$, and $25 \times$ in intensity. Eleven of these are Type 1 XUVs by the T07 definition: NGC 4117, NGC 3073, NGC 5338, NGC 3522, NGC 5173, NGC 7077, NGC 7360, UGC 6003, UGC 7020A, IC 1024, and NGC 3156. Eight of these are also UV-Bs: NGC 3073, NGC 5173, NGC 7077, UGC 6003, UGC 6805, UGC 7020A, NGC 3773, and IC 1024.

(A color version of this figure is available in the online journal.)

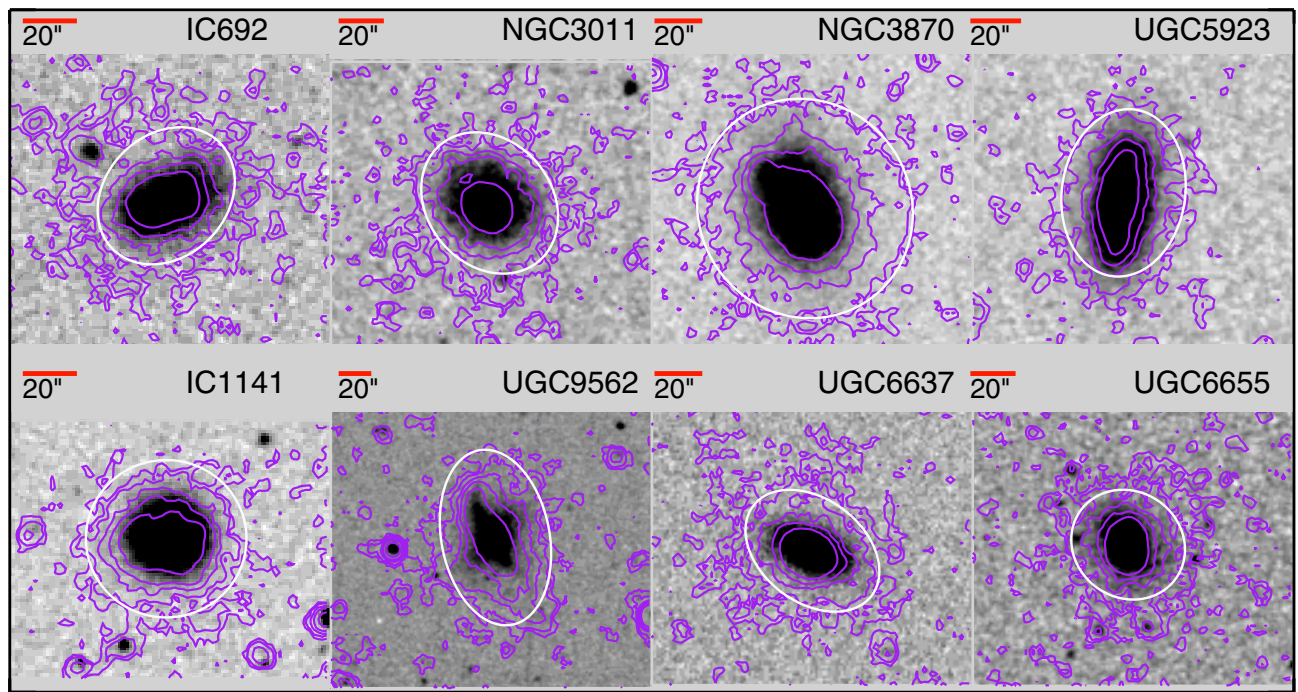

Figure 9. Smoothed NUV contours (purple) overlaid on DSS-II red images of UV-B disk E/S0s that are not also XUV-disk E/S0s. $R_{\mathrm{UVSF}}$ is indicated in white, and contours start at $\sim 28.6 \mathrm{AB}$ mag arcsec ${ }^{-2}$ and go up by $2 \times, 5 \times, 10 \times$, and $25 \times$ in intensity. None of these are Type 1 XUVs by the T07 definition. Some galaxies where the contours show extent beyond $R_{\mathrm{UVSF}}$ do not pass the test that this emission is $>3 \sigma$ above the PSF shelf (see Section 3.2.2).

(A color version of this figure is available in the online journal.) 


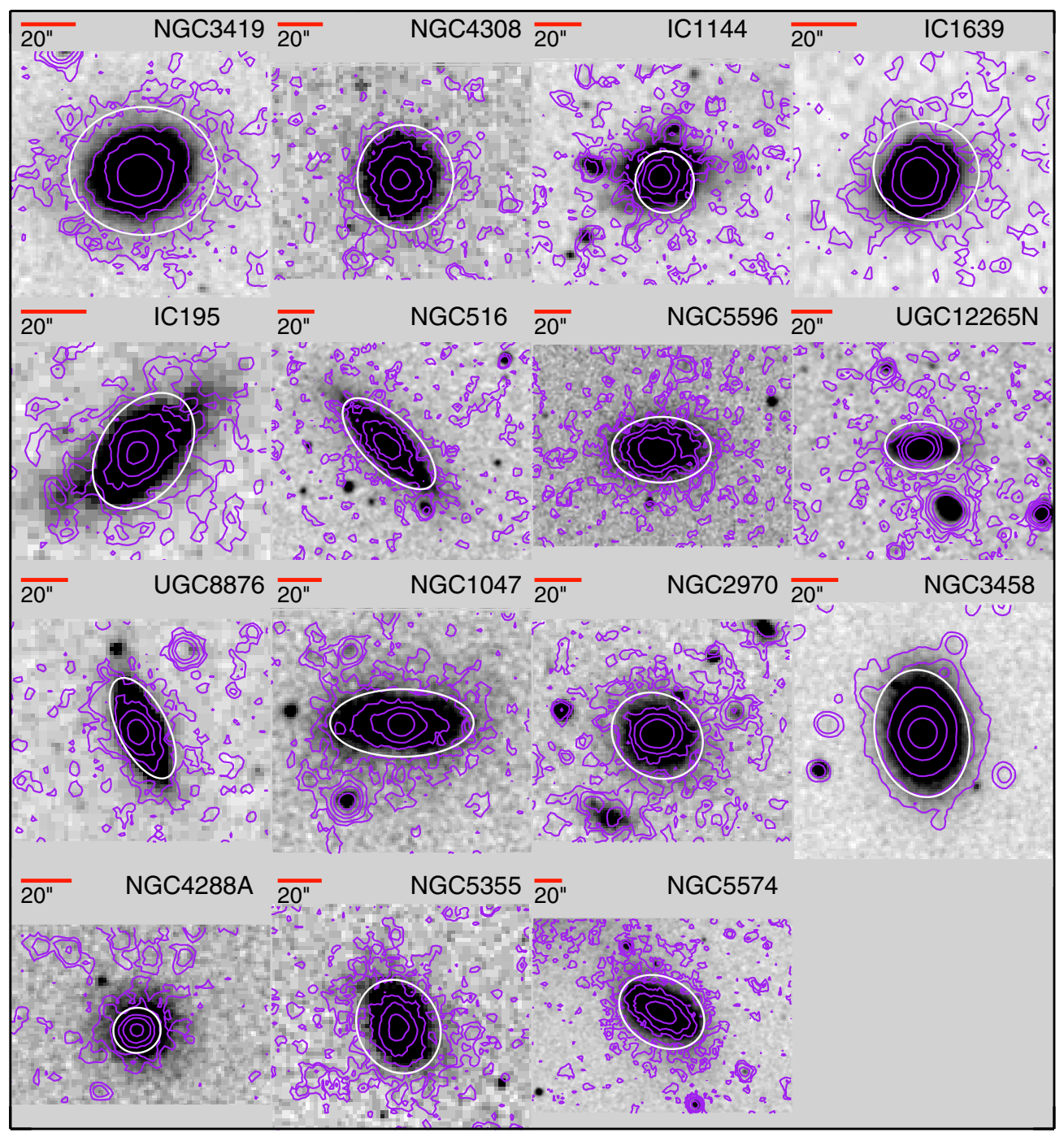

Figure 10. Smoothed NUV contours (purple) overlaid on DSS-II red images of E/S0s in our sample without XUV or UV-B disks. $R_{\mathrm{UVSF}}$ is indicated in white, and contours start at $\sim 28.6 \mathrm{AB}$ mag $\operatorname{arcsec}^{-2}$ and go up by $2 \times, 5 \times, 10 \times$, and $25 \times$ in intensity. Five of these are Type 1 XUVs by the T07 definition: NGC 3419 , NGC 4308, IC 195, UGC 8876, and NGC 5355. Some galaxies where the contours show extent beyond $R_{\mathrm{UVSF}}$ do not pass the test that this emission is $>3 \sigma$ above the PSF shelf (see Section 3.2.2).

(A color version of this figure is available in the online journal.)

impact on the appearances/properties of early types than late types, where they may be obscured by generally higher levels of star formation.

\subsection{Ubiquity of XUVs Compared to UV-Bs}

The widespread distribution of the XUV disks in color and stellar mass seems to suggest an association with evolutionary processes affecting the galaxy population broadly. A potential scenario for creating extended, star-forming disks around early types is external acquisition of extended gas, whether delivered by companion interactions or fresh cosmic gas accretion, and subsequent conversion of this gas to stars. In early-type galaxies, such extended disks or rings of $\mathrm{H}$ I are frequently observed and often believed to be associated with external accretion (e.g., Sage \& Welch 2006; Morganti et al. 2006; Oosterloo et al. 2007, 2010).

T07 find that $\sim 75 \%$ of their Type 1 XUV disks show evidence for interactions or minor perturbations. An interaction scenario could explain the widespread demographics of XUVs in our E/S0s, especially since the XUVs we identify using the original
T07 definition, which favors discovery of the weakest XUVs, are the most broadly distributed (Figure 5). To confirm such an association, more complete knowledge of the companion statistics of our sample would be needed.

For UV-B disks, which appear to have a somewhat massdependent distribution, the higher UV-B disk frequency at low masses (below $M_{\mathrm{t}}$ ) could hint at a gas delivery mechanism with a preferred mass scale, as in the cold-accretion scenario (e.g., Birnboim \& Dekel 2003; Kereš et al. 2005, 2009; Dekel $\&$ Birnboim 2006). However, an in-depth examination of the environments and group properties of a larger, statistical sample of such galaxies will be necessary to distinguish between various scenarios for producing extended star formation in early types.

\subsection{Relationship to Star Formation and H I Content}

An important question to ask about the apparently young UV disks we observe around E/S0s is: are they actually associated with substantial disk growth?

The higher $(\sim 40 \%)$ frequency of XUVs in both red- and blue-sequence E/S0s versus in late types seems to link XUV 
disks to a galaxy population associated with weak or inefficient star formation. Moreover, our E/S0 XUV disks do not show an association with blue optical outer-disk colors nor with enhanced H I content (see Section 4.3). The association of XUV disks with weak or inefficient star formation is consistent with the observation of a high $(\sim 70 \%)$ rate of XUV disks in massive optically low surface brightness galaxies, which are known for inefficient star formation as well (Boissier et al. 2008). It is also consistent with T07's result associating lower SFR/M $M_{\mathrm{H}}$ with Type 1 XUV disks. In addition, simulations of XUV disks in spiral galaxies show that star formation in these objects can proceed for as long as $4 \mathrm{Gyr}$ without producing enough stars to create a high surface brightness optical component (Bush et al. 2008).

In contrast, although XUV and UV-B classifications overlap, our UV-B disk galaxies as a class are characterized by bluer optical outer-disk colors and larger reservoirs of $\mathrm{H}$ I gas than E/S0s without UV-B disks (see Section 5.3). Thus, UV-B disks are more closely associated with significant disk star formation than are XUV disks. In addition, if we consider the blue sequence below $M_{\mathrm{t}}$, where the numbers and properties of E/S0s suggest disk building is most active (KGB), we find all sample galaxies save one are classified as UV-B disks (Figure 5). This strong link between UV-B disks and the sub- $M_{\mathrm{t}}$ blue sequence seems to support the scenario of significant growth in the optical outer disks of E/SOs in this regime.

\section{CONCLUSIONS}

We have used UV, optical, and IR imaging to study extendeddisk star formation in a sample of 38 red- and blue-sequence $\mathrm{E} / \mathrm{S} 0$ s in the stellar mass regime below $\sim 4 \times 10^{10} M_{\odot}$ and in primarily field environments. We introduce two new classifications: a purely quantitative version of the XUV disk classification, akin to the Type 1 XUV definition of Thilker et al. (2007; T07); and an alternative $U V$-B disk classification, with NUV $-K$ color indicating $\gtrsim 10 \%$ young population in the outer optical disk beyond the $50 \%$ light radius. We summarize key results from the application of these classifications below.

1. We identify a high $61 \% \pm 9 \%$ combined frequency of XUV and UV-B disks. Since the classifications partially overlap, this frequency reduces to separate $39 \% \pm 9 \%$ and $42_{-8}^{+9} \%$ frequencies for XUV disks and UV-B disks, respectively. In the XUV-disk case, the observed frequency is approximately twice the $\sim 20 \%$ reported by T07 for primarily late-type galaxies, although differences in XUV-disk criteria and possible detection biases could affect this comparison.

2. UV colors of both XUV and UV-B disks typically imply $<1$ Gyr ages, and most of the identified UV disks extend beyond the optical $R_{25}$ radius.

3. XUV-disk host galaxies occupy a widespread distribution in color and stellar mass, while UV-B disks more strongly prefer the blue sequence and may also prefer the low-mass regime.

4. XUV disks appear to be associated with low-level star formation, whereas UV-B disks appear to be more clearly associated with significant star formation. UV-B disk galaxies are also closely linked to the population of blue-sequence $\mathrm{E} / \mathrm{S} 0 \mathrm{~s}$ in the stellar mass regime below the "gas-richness threshold mass" at $M_{\mathrm{t}} \sim 5 \times 10^{9} M_{\odot}$ (Kannappan et al. 2009; KGB), supporting the idea that such galaxies represent an actively disk-building population (KGB).
Our results suggest that XUV-disk formation could be related to a process that affects the galaxy population broadly, such as interactions, while UV-B disk formation could be related to a process with a mass-scale preference, such as cold-mode gas accretion. Existing data do not yet allow us to disentangle such effects in the E/S0 population, but the purely quantitative classifications we have developed in this work are well suited to application in larger statistical samples, which will allow us to construct a more complete picture of the local and global environments of star-forming E/SOs. In subsequent work, we plan to combine quantitative metrics of both disk building and environment in a large volume-limited survey in order to constrain the origin and future evolution of star-forming E/SOs and further probe the intriguing possibility of early-to-late-type transformation.

We thank S. Jogee for her role in acquiring the Spitzer data, M. Haynes for the early release of GALEX imaging of NGC 3773, and the anonymous referee for suggestions that motivated substantial improvements to this work. We also thank D. Stark for helpful conversations on the topic of refining data analysis codes. We thank C. Clemens, K. Eckert, A. Leroy, M. Norris, and L. Wei for useful discussions as well. A.J.M. acknowledges support from the NASA Harriett G. Jenkins Predoctoral Fellowship Program. This work uses observations made with the NASA Galaxy Evolution Explorer. GALEX is operated for NASA by Caltech under NASA contract NAS5-98034. We acknowledge support from the GALEX Guest Investigator program under NASA grant NNX07AT33G. This work uses observations made with the Spitzer Space Telescope, operated by the Jet Propulsion Laboratory, Caltech under a contract with NASA. Support for this work was also provided by NASA through an award issued by JPL/Caltech. This work uses observations from the SDSS; funding for the SDSS and SDSSII has been provided by the Alfred P. Sloan Foundation, the Participating Institutions, the National Science Foundation, the U.S. Department of Energy, the National Aeronautics and Space Administration, the Japanese Monbukagakusho, the Max Planck Society, and the Higher Education Funding Council for England. The SDSS Web site is http://www.sdss.org/.

Facilities: GALEX, Spitzer

\section{REFERENCES}

Abazajian, K. N., Adelman-McCarthy, J. K., Agüeros, M. A., et al. 2009, ApJS, 182,543

Barnes, J. E. 2002, MNRAS, 333, 481

Bertin, E., \& Arnouts, S. 1996, A\&AS, 117, 393

Birnboim, Y., \& Dekel, A. 2003, MNRAS, 345, 349

Boissier, S., Gil de Paz, A., Boselli, A., et al. 2008, ApJ, 681, 244

Bruzual, G., \& Charlot, S. 2003, MNRAS, 344, 1000

Bush, S. J., Cox, T. J., Hernquist, L., Thilker, D., \& Younger, J. D. 2008, ApJ, 683, L13

Cardelli, J. A., Clayton, G. C., \& Mathis, J. S. 1989, ApJ, 345, 245

Cortese, L., \& Hughes, T. M. 2009, MNRAS, 400, 1225

Davies, R. L., Efstathiou, G., Fall, S. M., Illingworth, G., \& Schechter, P. L. 1983, ApJ, 266, 41

Dekel, A., \& Birnboim, Y. 2006, MNRAS, 368, 2

Donovan, J. L., Serra, P., van Gorkom, J. H., et al. 2009, AJ, 137, 5037

Emsellem, E., Cappellari, M., Krajnović, D., et al. 2007, MNRAS, 379, 401

Gil de Paz, A., Madore, B. F., Boissier, S., et al. 2005, ApJ, 627, L29

Gil de Paz, A., Madore, B. F., Boissier, S., et al. 2007, ApJ, 661, 115

Governato, F., Willman, B., Mayer, L., et al. 2007, MNRAS, 374, 1479

Hibbard, J. E., \& Mihos, J. C. 1995, AJ, 110, 140

Jansen, R. A., Fabricant, D., Franx, M., \& Caldwell, N. 2000a, ApJS, 126, 331 Jansen, R. A., Franx, M., Fabricant, D., \& Caldwell, N. 2000b, ApJS, 126, 271 Kannappan, S. J. 2004, ApJ, 611, L89 
Kannappan, S. J., Guie, J. M., \& Baker, A. J. 2009, AJ, 138, 579 (KGB)

Kannappan, S. J., \& Wei, L. H. 2008, in AIP Conf. Ser. 1035, The Evolution of Galaxies Through the Neutral Hydrogen Window, ed. R. Minchin \& E. Momjian (Melville, NY: AIP), 163

Kauffmann, G., Heckman, T. M., Budavri, T., et al. 2007, ApJS, 173, 357

Kauffmann, G., Heckman, T. M., White, S. D. M., et al. 2003, MNRAS, 341, 54

Kereš, D., Katz, N., Fardal, M., Davé, R., \& Weinberg, D. H. 2009, MNRAS, 395,160

Kereš, D., Katz, N., Weinberg, D. H., \& Davé, R. 2005, MNRAS, 363, 2

Lemonias, J. J., Schiminovich, D., Thilker, D., et al. 2011, ApJ, 733, 74

Leroy, A. K., Walter, F., Brinks, E., et al. 2008, AJ, 136, 2782

Marino, A., Rampazzo, R., Bianchi, L., et al. 2011, MNRAS, 411, 311

Moffett, A. J., Kannappan, S. J., Laine, S., et al. 2010, in ASP Conf. Ser. 423, Galaxy Wars: Stellar Populations and Star Formation in Interacting Galaxies, ed. B. J. Smith, N. Bastian, S. J. U. Higdon, \& J. L. Higdon (San Francisco, CA: ASP), 346

Morganti, R., de Zeeuw, P. T., Oosterloo, T. A., et al. 2006, MNRAS, 371, 157

Morrissey, P., Conrow, T., Barlow, T. A., et al. 2007, ApJS, 173, 682
Naab, T., Jesseit, R., \& Burkert, A. 2006, MNRAS, 372, 839

O’Connell, R. W. 1999, ARA\&A, 37, 603

Oosterloo, T., Morganti, R., Crocker, A., et al. 2010, MNRAS, 409, 500

Oosterloo, T. A., Morganti, R., Sadler, E. M., van der Hulst, T., \& Serra, P. 2007, A\&A, 465, 787

Paturel, G., Petit, C., Prugniel, P., et al. 2003, A\&A, 412, 45

Sage, L. J., \& Welch, G. A. 2006, ApJ, 644, 850

Salim, S., \& Rich, R. M. 2010, ApJ, 714, L290

Salpeter, E. E. 1955, ApJ, 121, 161

Schlegel, D. J., Finkbeiner, D. P., \& Davis, M. 1998, ApJ, 500, 525

Steinmetz, M., \& Navarro, J. F. 2002, New Astron., 7, 155

Thilker, D. A., Bianchi, L., Boissier, S., et al. 2005, ApJ, 619, L79

Thilker, D. A., Bianchi, L., Meurer, G., et al. 2007, ApJS, 173, 538 (T07)

Thilker, D. A., Bianchi, L., Schiminovich, D., et al. 2010, ApJ, 714, L171

Wei, L. H., Kannappan, S. J., Vogel, S. N., \& Baker, A. J. 2010, ApJ, 708, 841

Wyder, T. K., Martin, D. C., Schiminovich, D., et al. 2007, ApJS, 173, 293

Yang, Y., Zabludoff, A. I., Zaritsky, D., \& Mihos, J. C. 2008, ApJ, 688, 945

Zaritsky, D., \& Christlein, D. 2007, AJ, 134, 135 